\title{
VI. Finanzausgleich
}

\section{Fehlanreize im Fiskalföderalismus}

Der Finanzausgleich ist zweifellos der neuralgische Punkt jedes föderativen Staates. Die Verteilung der Gelder auf die staatlichen Ebenen folgt dabei nicht rationalen Kriterien, sondern ist das Ergebnis eines ständigen Machtkampfs. Das suboptimale Zusammenspiel der föderalen Ebenen erklärt zum Teil die Entwicklung der Staatsverschuldung. So war die chronische Unterfinanzierung des Kaiserreichs nicht zuletzt auf seine Struktur als „Bund der deutschen Fürsten“ beziehungsweise als Bund der Einzelstaaten zurückzuführen ${ }^{1}$. Die Finanzverfassung des ersten deutschen Nationalstaats basierte auf einem Trennsystem, in dem das Reich nur „Kostgänger der Einzelstaaten“ war. Im kongenialen Zusammenspiel mit dem Reichstag sorgten die Einzelstaaten dafür, dass das Problem der Reichsfinanzen weder durch den Übergang zur Hochschutzzollpolitik ${ }^{2}$ noch durch die Erhebung von Matrikularbeiträgen gelöst wurde. Die ausdrücklich in der Verfassung vorgesehene Möglichkeit, direkte Reichssteuern zu erheben, wurde vom Bundesrat ebenfalls konterkariert. Zwar gelang dem Reich mit der Erbschaftsteuer von 1906 und der drei Jahre später eingeführten Steuer auf Dividenden- und Zinserträge erstmalig der Einbruch in die Domäne der direkten Steuern, doch war dies finanziell von keiner großen Relevanz ${ }^{3}$. Bedeutsamer waren die kurz vor Kriegsausbruch und insbesondere im Krieg einmalig erhobenen

1 Vgl. den Überblick bei Carsten Burhop, Wirtschaftsgeschichte des Kaiserreichs 1871-1918, Göttingen 2011, S.81-99, sowie Hans Fenske, Deutsche Verfassungsgeschichte vom Norddeutschen Bund bis heute, Berlin ${ }^{2}$ 1984, S. 18.

${ }^{2}$ Durch die Lex Franckenstein wurde dem Reich auferlegt, alle Zollerträge, die 130 Millionen Mark überschritten, den Einzelstaaten zu überweisen. Vgl. Eckart Schremmer, Steuern und Staatsfinanzen während der Industrialisierung Europas. England, Frankreich, Preußen und das Deutsche Reich 1800 bis 1914, Berlin u.a. 1994, S.178ff., sowie Jürgen von Kruedener, The Franckenstein Paradox in the Intergovernmental Fiscal Relations of Imperial Germany, in: Peter-Christian Witt (Hrsg.), Wealth and Taxation in Central Europe. The History and Sociology of Public Finance, Leamington Spa u. a. 1987, S. 111-123.

${ }^{3}$ Vgl. allgemein Peter-Christian Witt, Die Finanzpolitik des Deutschen Reiches von 1903 bis 1913. Eine Studie zur Innenpolitik des Wilhelminischen Deutschland, Lübeck/ Hamburg 1970, sowie Wilhelm Gerloff, Die Finanz- und Zollpolitik des Deutschen Reiches nebst ihren Beziehungen zu Landes- und Gemeindefinanzen von der Gründung des Norddeutschen Bundes bis zur Gegenwart, Jena 1913. 
Reichssteuern ${ }^{4}$. Niall Ferguson hat die These aufgestellt, dass das Reich aufgrund seiner chronischen Unterfinanzierung den Rüstungswettlauf zu verlieren drohte und daher den Ersten Weltkrieg forciert habe ${ }^{5}$. Diese finanzpolitische Variante der Flucht in die Außenpolitik verdeutlicht zwar die schwierige Lage der Reichsfinanzen und besitzt insofern heuristischen Wert, überschätzt aber die Bedeutung finanzpolitischer Argumente für politische Entscheidungsträger.

Nach der Niederlage von 1918 kam es mit der Finanzreform von Matthias Erzberger zu einer Neuordnung der deutschen Finanzverfassung - eine Neuordnung, die eine tiefe Zäsur markierte und auch als Reaktion auf die missliche Situation des Kaiserreichs verstanden werden muss. „Die mangelhafte Ausstattung des alten Reiches mit Steuern“ war für den Reichsfinanzminister „der schwächste Punkt“ der Reichsverfassung von $1871^{6}$. Erzberger nutzte die Ausnahmesituation der Jahre 1919/20 daher konsequent, um die Reichsfinanzen zu stärken und die Länder- und Kommunalfinanzen zu schwächen ${ }^{7}$. Wörtlich führte er aus:

„In den Trümmern des Krieges muß nach Neuland gesucht werden. So vieles, fast alles ist anders geworden. [...] Wo ist hier Neuland für die Reichsfinanzen zu gewinnen? Der große Steuersouverän der Zukunft kann nur das einige Deutsche Reich sein [...]. Dieses kostbare Gut unserer Väter, der deutsche Nationalstaat, [...] muß leben und sich entwickeln können. Dazu braucht das Reich nicht nur Geld, sondern auch ein neues System der Steuerordnung. ${ }^{8}$

Erzbergers unitarischen Ansatz umsetzend, wies die Weimarer Reichsverfassung dem Reich die Gesetzgebungshoheit über einen Großteil der Steuern zu. In Berlin hatte man lediglich auf die „Erhaltung der Lebensfähigkeit der Länder Rücksicht zu nehmen “9. Mit dem Trennsystem des Kaiserreichs

${ }^{4} 1913$ wurde ein „Wehrbeitrag“, 1916 eine „Kriegssteuer“ und 1918 eine „außerordentliche Kriegsabgabe“ erhoben. Vgl. RGBl. 1913, S.505-521; RGBl. 1916, S. 561-572; RGBl. 1918, S. 964-974.

${ }^{5}$ Vgl. Niall Ferguson, Public finance and national security: The domestic origins of the First World War revisited, in: Past and Present 142 (1994), S. 141-168, sowie Ferguson, Krieg, S. 143-187.

${ }^{6}$ Erzberger, Reden, S. 111.

${ }^{7}$ Vgl. Josef Wysocki, Die Kommunalfinanzen in Erzbergers Reformkonzept: Finanzzuweisungen statt eigener Steuern, in: Karl-Heinrich Hansmeyer (Hrsg.), Kommunale Finanzpolitik in der Weimarer Republik, Stuttgart 1973, S. 35-59, hier S. 45 f.

${ }^{8}$ Erzberger, Reden, S. 7 .

${ }^{9}$ Artikel 8 der Verfassung des Deutschen Reichs vom 11.8. 1919, hrsg. von Hermann Mosler, Stuttgart 1988, S.6f. Vgl. auch Artikel 11 der Weimarer Reichsverfassung sowie das Landessteuergesetz vom 30.3. 1920, in: RGBl. 1920, S. 402-416. 
brechend, wurde ein in seinen Grundzügen bis heute geltendes Verbundsystem eingeführt. Das Reich wies nunmehr den Ländern die Gelder zu. Reichsüberweisungssteuern mit festen Quotenbeteiligungen ersetzten die Matrikularbeiträge der Länder an das Reich. Nun waren Länder und Kommunen die Kostgänger des Reichs. Zudem verloren die Einzelstaaten auch die Verwaltungskompetenz. Mit dem Jahr 1920 entstand die Reichsfinanzverwaltung $^{10}$.

Die (finanz)politische Macht der Länder wurde zunächst von Erzberger beschnitten und dann von den Nationalsozialisten gebrochen. Nach Kriegsende 1945 kam es jedoch zu einer Renaissance des Föderalismus, und es war kein Zufall, dass mit Hans Ehard ein Ministerpräsident - nämlich der bayerische - nach Herrenchiemsee einlud, um über die Grundzüge einer künftigen Verfassung zu beraten ${ }^{11}$. Bayern legte dabei einen Entwurf vor ${ }^{12}$, der auf die Bildung eines Staatenbunds hinauslief und die Zentrale wie im Kaiserreich durch Matrikularbeiträge in finanzpolitischer Abhängigkeit von den Ländern zu halten gedachte ${ }^{13}$.

Die Sozialdemokratie - insbesondere ihr Vorsitzender Kurt Schumacher ${ }^{14}$ - steuerte dagegen einen eher unitarischen, bewusst an Erzberger anknüpfenden Kurs ${ }^{15}$. Laut den Nürnberger Richtlinien der SPD vom Sommer 1947 war eine „einheitliche Finanzpolitik [...] notwendig, weil sie ein wesentliches Mittel zur Lenkung der Wirtschaft ist und weil der Neuaufbau eine gerechte

${ }^{10}$ Vgl. Herbert Leidel, Die Begründung der Reichsfinanzverwaltung, Bonn 1964, sowie Witt, Reichsfinanzminister und Reichsfinanzverwaltung, S. 41-61.

${ }^{11}$ Vgl. Der Parlamentarische Rat 1948-1949. Akten und Protokolle, Bd. 2: Der Verfassungskonvent auf Herrenchiemsee, bearb. von Peter Bucher, Boppard 1981, S. VII. Vgl. allgemein Michael F. Feldkamp, Der Parlamentarische Rat 1948-1949. Die Entstehung des Grundgesetzes, Göttingen 1998.

${ }^{12}$ Vgl. Parlamentarischer Rat, Bd. 2, S. LVI-LXIII und S. 1-52 (Dok. 1).

${ }^{13}$ So lautete Artikel 8, Absatz 2 des bayerischen Entwurfs (ebenda, S. 5): „Soweit diese Einnahmen [des Bundes] nicht ausreichen, sind die überschießenden Ausgaben durch Beiträge der Länder nach Maßgaben ihrer Leistungsfähigkeit zu decken. [...] Überschüsse der Bundeseinnahmen sind den Ländern nach den gleichen Grundsätzen zu überweisen. " Beinahe noch wichtiger war den bayerischen Vertretern allerdings, die Biersteuer in die alleinige Kompetenz der Länder übergehen zu lassen.

${ }^{14} \mathrm{Vgl}$. Wolfgang Benz, Der Verfassungskonvent von Herrenchiemsee, in: APuZ B 3233/98, S. 13-19, hier S. 15.

${ }^{15}$ So erklärte Otto Heinrich Greve (SPD): „Wir sind der Auffassung, daß wir, von gewissen Modifikationen abgesehen, wieder zu dem System zurückkehren sollten, das mit dem Namen Erzberger verknüpft ist. “ 7. Sitzung des Parlamentarischen Rats am 21.10.1948, in: Parlamentarischer Rat. Stenographische Berichte, Bonn 1948/49, S. 102 . 
Lastenverteilung verlangt ${ }^{\star 16}$. Die Schaffung einheitlicher Lebensverhältnisse und die Bewältigung der Nachkriegsprobleme waren weitere Argumente für eine Zentralisierung ${ }^{17}$. In der CDU herrschte eine starke Spannung zwischen Föderalisten und Zentralisten. Während Adenauer die Erzbergersche Finanzreform mit „Zentralismus und [...] Korruption“ gleichsetzte und die Ansicht vertrat, dass es "Sauberkeit und Ordnung" nur in einem Trennsystem gebe, führte sein Parteifreund Jakob Kaiser aus, „man müsse nicht nur die Frage nach den lebensfähigen Ländern, sondern nach einem lebensfähigen Gesamtdeutschland stellen ${ }^{\text {“18 }}$. Trotz dieser Konflikte fand der föderative Ansatz schließlich eine deutliche Mehrheit in der Union.

Doch das sollte im Parlamentarischen Rat nicht von ausschlaggebender Bedeutung sein, da die FDP in Fragen der Finanzverfassung mit der SPD stimmte, wobei die Formulierung treffender wäre, dass die Sozialdemokraten mit den Liberalen stimmten ${ }^{19}$. Es war insbesondere der Hermann HöpkerAschoff (FDP), der die Diskussion über den Abschnitt „Das Finanzwesen“ dominierte $^{20}$. Abermals beeinflusste ein preußischer Finanzminister - HöpkerAschoff hatte dieses Amt zwischen 1925 und 1931 bekleidet - die deutsche Finanzverfassung entscheidend. Doch Höpker-Aschoff stand nicht in der Tradition von Miquel, sondern in der von Erzberger. Er war bereits in der Weimarer Zeit ein entschiedener Verfechter des Einheitsstaats gewesen und hatte versucht, die Reichsreform über einen „Pakt zwischen Braun und Brüning“ durchzusetzen ${ }^{21}$. Sein auch bei den Liberalen nicht unumstrittener Unitarismus bestimmte seine Argumentation im Parlamentarischen Rat ${ }^{22}$.

${ }^{16}$ Richtlinien für den Aufbau der Deutschen Republik, in: Protokoll der Verhandlungen des Parteitags der Sozialdemokratischen Partei Deutschlands vom 29. Juni bis 2. Juli 1947 in Nürnberg, Hamburg o. J., S. 225 ff., hier S. 226.

${ }^{17}$ Vgl. Michael G. M. Antoni, Sozialdemokratie und Grundgesetz, Bd. 1: Verfassungspolitische Vorstellungen der SPD von den Anfängen bis zur Konstituierung des Parlamentarischen Rates 1948, Berlin 1991, S. 240.

${ }^{18}$ Die CDU/CSU im Parlamentarischen Rat. Sitzungsprotokolle der Unionsfraktion, bearb. von Rainer Salzmann, Stuttgart 1981, S.33f.

${ }^{19}$ Der SPD-Abgeordnete Dr. Greve erklärte: „Ich bin in der selten glücklichen Lage, für meine Freunde und mich zu erklären, daß wir den Ausführungen des Herrn Kollegen Dr. Höpker-Aschoff in ihren vollem Inhalt zustimmen." 7. Sitzung des Parlamentarischen Rats vom 21.10.1948, S. 101.

${ }^{20}$ Vgl. Frank Spieker, Hermann Höpker Aschoff. Vater der Finanzverfassung, Berlin 2004.

${ }^{21}$ Vgl. Hermann Höpker-Aschoff, Deutscher Einheitsstaat. Ein Beitrag zur Rationalisierung der Verwaltung, Berlin 1928, sowie Hagen Schulze, Otto Braun oder Preußens demokratische Sendung. Eine Biographie, Frankfurt a. M. u. a. 1977, S. 689-707.

${ }^{22}$ Vgl. allgemein Karl-Heinz Lamberty, Die Stellung der Liberalen zum föderativen Staatsaufbau in der Entstehungsphase der Bundesrepublik Deutschland 1945-1949, Diss., Bonn 1983. 
Als Berichterstatter für das Finanzwesen vermochte er die Diskussion vorzustrukturieren. Die Einkommensteuer den Ländern zu überlassen, lehnte Höpker-Aschoff $a b$, da unterschiedliche Steuersätze sozial ungerecht und ökonomisch belastend seien. Den Primat des Bundes bei der Steuergesetzgebung rechtfertigte der ehemalige Finanzminister mit einer Argumentation, die der sozialdemokratischen sehr ähnelte: „Die Finanzpolitik ist gleichzeitig Sozialpolitik, Wirtschafts- und Währungspolitik. ${ }^{(223}$ Sein ursprünglicher Plan, die Einkommen-, Körperschaft- und Umsatzsteuer in einem Topf zusammenzufassen und die Einnahmen qua Schlüsselzuweisungen auf Bund und Länder zu verteilen, scheiterte am Veto der Alliierten, genauso wie die Absicht, eine Bundesfinanzverwaltung aufzubauen ${ }^{24}$. Nach der Instrumentalisierung der Finanzpolitik durch den NS-Staat misstrauisch, fürchteten die Besatzungsmächte eine zu mächtige Zentrale und forderten eine stärkere Stellung der Länder ${ }^{25}$. Als Kompromiss wurde ausgehandelt, dass der Bund die Umsatzsteuer zur Gänze erhielt und über den Weg eines zustimmungspflichtigen Gesetzes auch am Aufkommen der Einkommen- und Körperschaftsteuer partizipieren konnte, falls der Haushalt anderweitig nicht auszugleichen $\operatorname{sei}^{26}$. Dies machte der Bund umgehend über sogenannte Inanspruchnahmegesetze geltend ${ }^{27} .1955$ wurde seine direkte Beteiligung an der Einkommensteuer im Grundgesetz verankert, die zunächst ein Drittel des Gesamtaufkommens betrug ${ }^{28}$. Die Einkommensteuer wurde also mit der ersten größeren Finanzreform integraler Bestandteil eines Steuerverbunds, der eine grundlegende Revision des von den Alliierten durchgesetzten Trennsystems darstellte ${ }^{29}$.

${ }^{23}$ Hermann Höpker-Aschoff, Schriftlicher Bericht zum Entwurf des Grundgesetzes für die Bundesrepublik Deutschland, in: Anlage zum Stenographischen Bericht der 9. Sitzung des Parlamentarischen Rats am 6.5.1949, S. 51-60, hier S. 54.

${ }^{24}$ Vgl. ebenda, S. 56 und S. 58. Das Bundesfinanzministerium fordert seit einigen Jahren, eine Bundessteuerverwaltung zu schaffen, und knüpft damit an die zwischen 1920 und 1945 bestehende Reichsfinanzverwaltung an, scheitert damit aber regelmäßig am Widerstand der Länder Vgl. Eike Alexander Senger, Die Reform der Finanzverwaltung in der Bundesrepublik Deutschland, Wiesbaden 2009, S. $222 \mathrm{f}$.

${ }^{25} \mathrm{Vgl}$. Hans-Jürgen Grabbe, Die deutsch-alliierte Kontroverse um den Grundgesetzentwurf im Frühjahr 1949, in: VfZ 26 (1978), S. 393-418.

${ }^{26}$ Vgl. Höpker-Aschoff, Bericht, S. 57, sowie Grundgesetz, Artikel 106, Absatz 3, Satz 1, in der Fassung vom 23.5. 1949, in: BGBl. 1949/50, I, S. 14.

${ }^{27}$ Vgl. Wolfgang Renzsch, Finanzverfassung und Finanzausgleich. Die Auseinandersetzungen um ihre politische Gestaltung in der Bundesrepublik Deutschland zwischen Währungsreform und deutscher Vereinigung (1948-1990), Bonn 1991, S. 75-132.

${ }^{28}$ Vgl. Gesetz zur Änderung und Ergänzung der Finanzverfassung (Finanzverfassungsgesetz) vom 23.12.1955, in: BGBl. 1955, I, S. 817f., hier S. 817 (\$1, Absatz 3).

${ }^{29}$ Vgl. u.a. Wilhelmine Dreißig, Zur Entwicklung der öffentlichen Finanzwirtschaft 
Mit der Finanzreform von 1969 wurde das Verbundsystem ausgebaut. Nach der Einkommensteuer wurde jetzt auch die Mehrwertsteuer zur Gemeinschaftsteuer. Zudem führte die Große Koalition eine Reihe von Mischfinanzierungen $\operatorname{ein}^{30}$ und intensivierte den horizontalen Finanzausgleich. Transfers zwischen den Ländern und Bundesmittel gleichen seitdem die unterschiedliche Finanzkraft der einzelnen Bundesländer weitgehend aus. Das Ziel besteht in der Schaffung einheitlicher beziehungsweise gleichwertiger Lebensverhältnisse $^{31}$.

Die Anfänge des horizontalen Finanzausgleichs gehen auf die Weimarer Republik zurück ${ }^{32}$. Forderungen, insbesondere zum Finanzausgleich zwischen armen und reichen Städten, waren bereits im Kaiserreich erhoben worden. Wissenschaftlich und politisch bereitete vor allem Johannes Popitz den kommunalen Finanzausgleich vor ${ }^{33}$. Je mehr das Ziel einheitlicher Lebensverhältnisse an Gewicht gewann, desto schwächer wurde der Föderalismus und desto stärker schwand die Finanz- und Entscheidungsautonomie der Länder und Kommunen.

Das Popitzsche Gesetz von der Anziehungskraft des zentralen Etats ${ }^{34}$ lässt sich nur bestätigen, wenn Länder- und Reichs- beziehungsweise Bundes-

seit dem Jahre 1950, in: Währung und Wirtschaft in Deutschland 1876-1975, hrsg. von der Deutschen Bundesbank, Frankfurt a. M. 1976, S.691-744, hier S. 734.

${ }^{30}$ Vgl. 21. Gesetz zur Änderung des Grundgesetzes (Finanzreformgesetz) vom 12.5. 1969, in: BGBl. 1969, I, S.359-362. Die Große Finanzreform von 1969 konnte erst nach einer harten Auseinandersetzung zwischen der Großen Koalition und der widerstrebenden Mehrheit der Bundesländer beschlossen werden. Vgl. Renzsch, Finanzverfassung, S. 219-260, sowie Ernst Heinsen, Der Kampf um die Große Finanzreform 1969, in: Rudolf Hrbek (Hrsg.), Miterlebt - Mitgestaltet. Der Bundesrat im Rückblick, Stuttgart 1989, S. 187-223.

${ }^{31}$ Vgl. den Überblick bei Stefan Korioth, Der Finanzausgleich zwischen Bund und Ländern, Baden-Baden 1997, S. 169-187.

${ }^{32}$ So versuchte das Landessteuergesetz, übergroße Disparitäten in der Finanzkraft zwischen den Ländern durch Zahlungen des Reichs auszugleichen. Zudem forderte das Landessteuergesetz von den Ländern, „für einen Lastenausgleich unter ihren Gemeinden und Gemeindeverbänden, insbesondere auf dem Gebiete der Armen-, Schul- und Polizeilasten, zu sorgen“. Landessteuergesetz vom 30.3.1920, in: RGBl. 1920, S. 402-416, hier S. 409 (\$33) und S. 413 (\$55).

${ }^{33}$ Vgl. Johannes Popitz, Der künftige Finanzausgleich zwischen Reich, Ländern und Gemeinden. Gutachten erstattet der Studiengesellschaft für den Finanzausgleich, Berlin 1932; vgl. auch Klaus Kinkel, Die Lehre von Popitz für die Gestaltung des gemeindlichen Finanzausgleichs und ihr Verhältnis zum gegenwärtigen Rechtszustand in der Bundesrepublik, Vergleich und Problematik unter besonderer Berücksichtigung einer kommunalen Finanzreform, Diss., Köln 1964.

${ }^{34}$ Vgl. Karl-Heinrich Hansmeyer, Das Popitzsche Gesetz von der Anziehungskraft des zentralen Etats, in: Herbert Timm/Heinz Haller (Hrsg.), Beiträge zur Theorie der öffent- 
einnahmen zusammengefasst werden. Beide Seiten weiteten ihre Anteile an den Steuereinnahmen seit 1920 auf Kosten der Kommunen aus ${ }^{35}$. Das Steueraufkommen der Kommunen war 1913 insbesondere aufgrund des Zuschlagsrechts auf die Einkommensteuer fast genauso hoch wie das des Reichs und weit höher als das der Einzelstaaten. In der Weimarer Republik sank der kommunale Anteil am Gesamtsteueraufkommen im Vergleich zum Kaiserreich nur leicht. Der große Einschnitt kam mit der Gründung der Bundesrepublik, als der Anteil der Kommunen auf nur noch 13 Prozent sank und bis heute auf diesem niedrigen Niveau blieb. In den 1950er und 1960er Jahren waren die Kommunen überhaupt nicht an der Einkommensteuer beteiligt. Im Rahmen der großen Finanzreform von $1969^{36}$ erhielten sie dann einen Einkommensteueranteil von zunächst 14 Prozent, der zehn Jahre später auf 15 Prozent erhöht wurde. Bund und Länder teilen sich seitdem das restliche Aufkommen der Einkommensteuer zur Hälfte. Für die stärkere Beteiligung des Bundes an der Einkommensteuer erhielten die Länder 1969 erstmalig nach dem Krieg einen Anteil an der Umsatzsteuer, die sich seither zum flexiblen Faktor der Finanzbeziehungen zwischen Bund und Ländern entwickelte. Beispielsweise erhielten die Länder 1995/96 einige Umsatzsteuerpunkte wegen der Einbeziehung der neuen Bundesländer in den Finanzausgleich sowie wegen der Neuregelung des Familienlastenausgleichs ${ }^{37}$.

Der Anteil der Kommunen an den gesamten öffentlichen Staatsausgaben sank im Laufe des 20.Jahrhunderts um acht Prozentpunkte, ging also deutlich weniger zurück als ihr Anteil am Steueraufkommen ${ }^{38}$. Obwohl die Kommunen zur Gebietskörperschaft mit dem geringsten Ausgabenanteil wurden, lag dieser am Ende des 20. Jahrhunderts immerhin bei einem knappen Viertel; ein Beleg für die „Systemfunktion“ der Kommunen ${ }^{39}$.

Die Zäsuren von 1919 und 1969 zeigen, dass die Entwicklung der Finanzverfassung und des Finanzausgleichs immer abhängig ist von der jeweiligen

lichen Ausgaben, Berlin 1967, S. 197-229, sowie Korioth, Finanzausgleich, insbesondere S. 187-197.

${ }^{35}$ Vgl. hierzu und zum Folgenden Marc Hansmann, Wege in den Schuldenstaat. Die strukturellen Probleme der deutschen Finanzpolitik als Resultat historischer Entwicklungen, in: VfZ 55 (2007), S. 425-461, hier S. 453.

${ }^{36}$ Vgl. Franz Klein, Die Finanzreform zwischen Bund, Ländern und Gemeinden, in: APuZ B 30/69, S. 3-23.

${ }^{37}$ Vgl. Ulrich Häde, Finanzausgleich. Die Verteilung der Aufgaben, Ausgaben und Einnahmen im Recht der Bundesrepublik Deutschland und der Europäischen Union, Tübingen 1996, S. 203 und S. 264.

${ }^{38}$ Vgl. hierzu und zum Folgenden Hansmann, Schuldenstaat, S. 454.

${ }^{39}$ Wilhelm Ribhegge, Die Systemfunktion der Gemeinden. Zur deutschen Kommunalgeschichte seit 1918, in: APuZ B 47/73, S. 3-29. 
Vorstellung, wie ein Staatswesen sinnvollerweise aufgebaut sein sollte. Nach dem Ersten Weltkrieg diente der Unitarismus als Orientierungsrahmen, während die Bundesrepublik zunehmend dem Modell eines unitarischen Bundesstaats folgte, das 1969 um das Leitbild eines kooperativen Föderalismus ergänzt wurde ${ }^{40}$. Ein Wettbewerbföderalismus mit großer Finanzautonomie sämtlicher Gebietskörperschaften würde nicht zum Ziel passen, gleichwertige Lebensverhältnisse zu schaffen; der kooperative Föderalismus mit seiner funktionalen Aufgabenverteilung zwischen den Gebietskörperschaften eignet sich dagegen ausgesprochen gut dafür ${ }^{41}$. Faktisch bedeutet diese Art des Föderalismus allerdings eine Zentralisierung der Staatstätigkeit, zumindest was die Handlungsspielräume von Ländern und Kommunen angeht ${ }^{42}$.

Die Länder verfügen spätestens seit der Finanzreform von 1969 über keine nennenswerten Gestaltungsmöglichkeiten auf der Einnahmenseite mehr, dafür ist aber jedes Steuergesetz im Bundesrat zustimmungspflichtig. Über den Länderfinanzausgleich wird dann noch die Finanzkraft weitestgehend ausgeglichen. Dieses System schafft Fehlanreize ${ }^{43}$. Parteipolitisch motivierte Blockadepolitik ist dabei noch nicht einmal das Kernproblem, auch wenn sie zum Beispiel Theo Waigels grundlegende Steuerreform ${ }^{44}$ und Hans Eichels Steuervergünstigungsabbaugeset ${ }^{45}$ verhinderte. Wesentlich problematischer

${ }^{40}$ Vgl. allgemein Konrad Hesse, Der unitarische Bundesstaat, Heidelberg 1962, sowie Gerhard Lehmbruch, Der unitarische Bundesstaat in Deutschland: Pfadabhängigkeit und Wandel, Köln 2002.

${ }^{41}$ Vgl. Ulrich Häde, Föderalismus und Einheitlichkeit der Lebensverhältnisse, in: Kai A. Konrad/Beate Jochimsen (Hrsg.), Föderalismuskommission II: Neuordnung von Autonomie und Verantwortung, Frankfurt a.M. u.a. 2008. S. 157-177; Wolfgang Renzsch, Der Streit um den Finanzausgleich. Die Finanzverfassung als Problem des Bundesstaates, in: Hans-Georg Wehling (Hrsg.), Die deutschen Länder. Geschichte, Politik, Wirtschaft, Wiesbaden ${ }^{3}$ 2004, S.373-395; Horst Zimmermann, Föderalismus und „Einheitlichkeit der Lebensverhältnisse“. Das Verhältnis regionaler Ausgleichsziele zu den Zielen des föderativen Staatsaufbaus, in: Kurt Schmidt (Hrsg.), Beiträge zu ökonomische Problemen des Föderalismus, Berlin 1987, S. 35-69.

${ }^{42}$ Charles B. Blankart (Die schleichende Zentralisierung der Staatstätigkeit: Eine Fallstudie, in: Zeitschrift für Wirtschafts- und Sozialwissenschaften 119 (1999), S.331-350, hier S.336) spricht mit Bezug auf die Bedeutung der Bundesgesetzgebung sogar von einer Entwicklung zum Einheitsstaat.

${ }^{43}$ Vgl. allgemein Jonathan A. Rodden, Hamilton's Paradox. The Promise und Peril of Fiscal Federalism, Cambridge 2006.

${ }^{44}$ Vgl. Reimut Zohlnhöfer, Die große Steuerreform 1998/99: Ein Lehrstück für Politikentwicklung bei Parteienwettbewerb im Bundestag, in: ZfParl 30 (1999), S. 326-345.

${ }^{45}$ Vgl. 19. Subventionsbericht. Bericht der Bundesregierung über die Entwicklung der Finanzhilfen des Bundes und der Steuervergünstigungen für die Jahre 2001-2004, hrsg. vom Bundesministerium der Finanzen, Berlin 2003, S. 29. 
ist, dass sich die Ministerpräsidenten mitunter mehr über die Bundespolitik profilieren als über ihre originären landespolitischen Aufgaben ${ }^{46}$. Unpopuläre Maßnahmen wie Steuererhöhungen oder Ausgabenreduzierungen im Sozialbereich sind im Bundesrat schwer durchzusetzen, während von Länderseite häufig Forderungen kommen, Sozialleistungen auszubauen. Kompromisse zwischen Bund und Ländern sind in der Regel teuer, und zwar insbesondere für den Bundeshaushalt. Mitunter hat sich der Bund Mehrheiten erkauft, vor allem durch die gezielte Förderung finanzschwacher, kleiner Bundesländer wie Bremen und Berlin ${ }^{47}$. Das Urteil von Philip Manow fällt daher eindeutig aus: „Die besondere Spielart des kooperativen Föderalismus begünstigte ,fiskalische Unverantwortlichkeit' der verschiedenen Staatsebenen", so dass im Gegensatz zur USA „die föderalen Staatsstrukturen nicht nur nicht hemmend, sondern sogar ausgabenexpansiv wirkten “48.

\section{Problematische Finanzierung der Länder}

Im Kaiserreich finanzierten sich die Einzelstaaten zu einem beträchtlichen Teil über die Gewinne der Eisenbahnen ${ }^{49}$. Zudem verfügten sie über die Gesetzgebungshoheit über die Einkommensteuer. 1919 wurde die Eisenbahn zur Reichsbahn und die Einkommensteuer zur Reichssteuer. Seitdem sind die Länder nur noch an den großen Steuern beteiligt, erhalten also faktisch Überweisungen von der Zentrale. Zutreffenderweise sprach man in der Weimarer Republik von „Reichsüberweisungssteuern“, während es heute „Gemeinschaftsteuern" heißt.

Die Länder verfügen über nur zwei Einnahmepositionen, deren Höhe sie selbst bestimmen können: die Grunderwerbsteuer und die Kreditaufnahme. Die Grunderwerbsteuer ist von ihrem Aufkommen her nicht signifikant; sie

\footnotetext{
${ }^{46}$ Vgl. allgemein Ursula Münch, Vom Gestaltungsföderalismus zum Beteiligungsföderalismus. Die Mitwirkung der Länder an der Bundespolitik, in: Wehling (Hrsg.), Länder, S. 355-371.

${ }^{47}$ Vgl. Renzsch, Finanzausgleich, S. 390.

${ }^{48}$ Philip Manow, Federalism and the Welfare State: The German Case, Bremen 2004, S. 2 .

${ }^{49}$ Vgl. Rainer Fremdling, Freight Rates and State Budget: the Role of the National Prussian Railways 1880-1913, in: Journal of European Economic History 9 (1980), S.21-39, insbesondere S.31-34; Mark Hallerberg, The political economy of taxation in Prussia, 1871-1914, in: Jahrbuch für Wirtschaftsgeschichte 2002/2, S. 11-33, hier S.17; Peter-Michael Prochnow, Staat im Wachstum. Versuch einer finanzwirtschaftlichen Analyse der preußischen Haushaltsrechnungen 1871-1913, Bd. 1, Münster 1977, S. 29 und S. 35; Spoerer, Evolution, S. $127 \mathrm{f}$.
} 
trägt weniger als zwei Prozent zur Finanzierung der Landeshaushalte bei ${ }^{50}$. Hingegen betrug 2009 die Kreditfinanzierungsquote in Niedersachsen neun Prozent und in Nordrhein-Westfalen elf Prozent ${ }^{51}$. In absoluten Zahlen waren das 2,3 und 5,6 Milliarden Euro. Der Verfassungsgerichtshof von Nordrhein-Westfalen verbot der neu gewählten Landesregierung im März 2011, die politisch gewünschten Mehrausgaben über eine milliardenschwere Erhöhung der ohnehin eklatanten Neuverschuldung zu finanzieren. Diese präzedenzlose Entscheidung verdeutlicht, dass die Länder ihre einzige nennenswerte Einnahmenquelle nicht mehr unbegrenzt ausschöpfen dürfen. Insbesondere Nordrhein-Westfalen hatte in den vergangenen Jahrzehnten hohe Finanzierungslücken. So ist die Verschuldung des Landes zwischen 1980 und 2010 von 15 Milliarden auf mindestens 128 Milliarden Euro gestiegen ${ }^{52}$. Ähnlich hohe Kreditfinanzierungsquoten, die unweigerlich zu einer hohen Steigerung der Verschuldung führten, wies Hessen auf. Dort stieg die Verschuldung zwischen 1970 und 2010 von 1,4 auf 39 Milliarden Euro ${ }^{53}$.

Die Länder haben sich unterschiedlich stark verschuldet - eine Tatsache, die nur zum geringen Teil auf das unterschiedlich hohe Steueraufkommen zurückgeführt werden kann $^{54}$. Abgeleitet aus dem im Grundgesetz verankerten Ziel der Gleichwertigkeit der Lebensverhältnisse ist ein Länderfinanzausgleich entstanden, der - aufgefüllt durch die Bundesergänzungszuweisungen - dafür sogt, dass jedes Bundesland 2008 mindestens 97,4 Prozent der durchschnittlichen Finanzkraft aufweisen konnte. Die Schuldenaufnahme ist daher in erster Linie das Resultat der Ausgabenhöhe. Länder, die sich bei den Ausgaben zurückhalten und hart gespart haben wie zum Beispiel MecklenburgVorpommern, Sachsen und Bayern vermochten daher, den Haushalt aus-

\footnotetext{
${ }^{50}$ In Nordrhein-Westfalen betrug das Aufkommen der Grunderwerbsteuer 2010 eine knappe Milliarde Euro, in Niedersachsen gut 0,3 Milliarden Euro. Vgl. Haushaltsplan 2010 des Landes Nordrhein-Westfalen, Kapitel 20.010, sowie Haushaltplan 2010 des Landes Niedersachsen, Einzelplan 13, Kapitel 1301, S.6. Niedersachsen hat die Grunderwerbsteuer mit Wirkung zum 1.1.2011 von 3,5 auf 4,5 Prozent erhöht.

${ }^{51}$ Vgl. Jahrbuch für öffentliche Finanzen 2010, S. 112 und S. 123 (eigene Berechnung).

${ }^{52}$ Vgl. Finanzplanung 2008 bis 2012 mit Finanzbericht 2009 des Landes NordrheinWestfalen, hrsg. vom Finanzministerium des Landes Nordrhein-Westfalen, Düsseldorf 2008, S. 54.

${ }^{53}$ Vgl. Die Einführung der Schuldenbremse in Hessen. Hintergründe und Argumente, hrsg. vom Hessischen Ministerium der Finanzen, Wiesbaden 2010, S. 7.

${ }^{54}$ Beispielsweise weist das hoch verschuldete Hessen nach dem Länderfinanzausgleich die höchste Finanzkraft auf. Vgl. Horst Zimmermann/Klaus-Dirk Henke/ Michael Broer, Finanzwissenschaft, München ${ }^{10} 2009$, S. 224; die folgende Angabe findet sich ebenda.
} 
zugleichen und sogar Überschüsse zu erwirtschaften ${ }^{55}$. Dagegen stehen mit Nordrhein-Westfalen, Hessen und Niedersachsen Länder, denen seit Jahrzehnten kein Haushaltsausgleich gelungen ist. Auch das Saarland und Bremen kommen trotz (oder wegen) des 1992 vom Bundesverfassungsgericht durchgesetzten Bailout $t^{56}$ nicht ohne eine hohe Nettoneuverschuldung aus ${ }^{57}$. Wegen überdurchschnittlicher Kreditfinanzierungs- und Zins-Ausgaben-Quoten hatte das Bundesverfassungsgericht für beide Länder eine „extreme Haushaltsnotlage" festgestellt und ihnen Sanierungshilfen in Form von SonderBundesergänzungszuweisungen zugesprochen, die von 1994 bis 2004 6,6 Milliarden Euro für das Saarland und über 8,5 Milliarden Euro für Bremen betrugen $^{58}$.

Die Haushaltssituation hat sich jedoch in beiden Ländern „nicht nachhaltig gebessert ${ }^{459}$. Die Wirkung des Finanzausgleichs besteht im Gegenteil darin, „unsolide Haushaltspolitik zu begünstigen “600. Die Landesregierungen werden nicht motiviert, „es besser als die jeweils anderen zu machen“. Immerhin bekräftigte das Bundesverfassungsgericht die aus dem „bündischen Prinzip“ abgeleitete Einstandspflicht im Jahr 2006 nicht, sondern verweigerte Berlin die geforderten Finanzhilfen. Insgesamt hat allerdings kein Land so viel Geld aus dem Finanzausgleich erhalten wie die Bundeshauptstadt. Immerhin vermochte Berlin zwischenzeitlich, den Haushalt auszugleichen ${ }^{61}$.

Mit einem Anteil von etwa 40 Prozent an den Gesamtausgaben schlagen in den Ländern die Personalkosten am stärksten zu Buche ${ }^{62}$. Allein NordrheinWestfalen hat 2010 knapp 21 Milliarden Euro dafür ausgegeben ${ }^{63}$. Dabei sind

${ }^{55}$ Vgl. Jahrbuch für öffentliche Finanzen 2010, S. 103 (Mecklenburg-Vorpommern), S. 155 (Sachsen) und S. 49 (Bayern).

${ }^{56}$ Ein Bailout ist die Schuldenübernahme beziehungsweise Hilfe durch Dritte, um eine Insolvenz zu vermeiden.

${ }^{57}$ Vgl. Jahrbuch für öffentliche Finanzen 2010, S. 69 (Bremen) und S. 145 (Saarland).

${ }^{58}$ Vgl. Kai A. Konrad/Holger Zschäpitz, Schulden ohne Sühne? Warum der Absturz der Staatsfinanzen uns alle betrifft, München 2010, S. 187.

${ }^{59}$ Ebenda, S. 188. Vgl. Charles B. Blankart, Föderalismus in Deutschland und Europa, Baden-Baden 2007, S. 142-149.

${ }^{60}$ Stefan Homburg, Anreizwirkungen des deutschen Finanzausgleichs, in: Finanzarchiv N.F. 51 (1994), S.312-330, hier 326; das folgende Zitat findet sich ebenda, S. 325.

${ }^{61}$ Vgl. Jahrbuch für öffentliche Finanzen 2010, S. 58.

${ }^{62}$ Vgl. Finanzplanung 2008 bis 2012 mit Finanzbericht 2009 des Landes NordrheinWestfalen, hrsg. vom Finanzministerium des Landes Nordrhein-Westfalen, Düsseldorf 2008, A 76.

${ }^{63}$ Vgl. hierzu und zum Folgenden Finanzplanung 2009 bis 2013 mit Finanzbericht 2010 des Landes Nordrhein-Westfalen, hrsg. vom Finanzministerium des Landes Nordrhein-Westfalen, Düsseldorf 2009, S. 32 und S. 34 . 
die Hochschulen nicht einmal mit eingerechnet, da sie rechtlich selbstständig sind und nur noch Zuschüsse erhalten. Das bevölkerungsreichste Bundesland beschäftigt 154000 Lehrer, 55000 Polizisten, 32000 Richter, Staatsanwälte und sonstige Justizangestellte sowie 29000 Finanzbeamte. Genau hier schlummert eine Zeitbombe. Während die Bundesverwaltung vergleichsweise klein ist und viele Städte nur in streng hoheitlichen Aufgabengebieten verbeamten, verfügen die Länder über riesige Beamtenapparate in den Bereichen Bildung, Wissenschaft, Polizei und Justiz. Zwar spart die öffentliche Hand bei Beamten Milliarden an jährlichen Versicherungsbeiträgen. Dafür müssen die Pensionen aber aus dem laufenden Haushalt finanziert werden.

Die drohende Kostenexplosion hat im Wesentlichen drei Gründe. In den 1970er Jahren hat die öffentliche Hand ihren Personalkörper stark ausgeweitet. Diese Jahrgänge gehen langsam aber sicher in die Pension, und das ist für die Länder sehr teuer, weil Lehrer, Professoren und Richter in hohen Besoldungsstufen eingruppiert sind. Zudem liegt das Pensionsniveau deutlich über dem Rentenniveau, da es bisher bei der Beamtenversorgung keine harten Kürzungen gegeben hat. Wie groß die drohende Gefahr für die Länderhaushalte ist, hat der Finanzwissenschaftler Stefan Homburg 2004 für das Land Niedersachsen ausgerechnet ${ }^{64}$. Bis 2030 steigt dort die Anzahl der Pensionäre von ungefähr 70000 im Jahr 2007 auf 110000 . Wenn der Haushalt nicht nachhaltig konsolidiert wird, droht sich im gleichen Zeitraum das Verhältnis von Pensions- und Zinsausgaben zu den laufenden Einnahmen von 24 Prozent auf 54 Prozent zu verschlechtern.

Hinweise auf die Höhe der Pensionsverpflichtungen geben die Eröffnungsbilanzen von Hamburg und Hessen. Hamburg ist das erste Bundesland, das auf eine kaufmännische Buchführung umgestellt hat und daher diese Zahlen ausweisen muss ${ }^{65}$. Die Hansestadt hatte 2006 Kreditmarktschulden in Höhe von rund 24 Milliarden Euro und Pensionsrückstellungen von 18 Milliarden Euro. Diese Rückstellungen haben ebenfalls den Charakter von Schulden, da hier Ansprüche von Privatpersonen an den Staat bestehen. Die 18 Milliarden Euro Pensionsrückstellungen werden der Transparenz halber ausgewiesen, liegen also nicht auf irgendeinem Konto oder in einem Fonds, wie es

\footnotetext{
${ }^{64}$ Vgl. Stefan Homburg, Nachhaltige Finanzpolitik für Niedersachsen, Hannover 2005; vgl. auch Tobias Benz/Christian Hagist/Bernd Raffelhüschen, Ausgabenprojektion und Reformszenarien der Beamtenversorgung in Niedersachsen. Studie im Auftrag des Bundes der Steuerzahler Niedersachsen und Bremen, Hannover 2009.

${ }^{65}$ Vgl. hierzu und zum Folgenden Hamburg zieht Bilanz. Geschäftsbericht zur Eröffnungsbilanz auf den 1. Januar 2006, hrsg. von der Freien und Hansestadt Hamburg, Hamburg 2006.
} 
ein Unternehmen machen müsste. Für Rückstellungen gigantische Kredite aufzunehmen, wäre allerdings betriebswirtschaftlich unsinnig. Hamburg wies ein Vermögen von 50 Milliarden Euro aus. Die landeseigenen Unternehmen und Beteiligungen wurden dabei mit knapp zehn Milliarden Euro bewertet. Abzüglich des Fremdkapitals blieb bei der Eröffnungsbilanz Hamburgs ein Eigenkapital von vier Milliarden Euro übrig. Das entspricht einer Eigenkapitalquote von nur acht Prozent. Im Zuge der Wirtschaftskrise von 2008/09 ist das Eigenkapital der Hansestadt Hamburg, deren Bewohner eines der höchsten Pro-Kopf-Einkommen Europas aufweisen, vollständig aufgezehrt worden ${ }^{66}$. Hessen wies in seiner im November 2009 vorgelegten Eröffnungsbilanz ein negatives Eigenkapital von 58 Milliarden Euro auf $f^{67}$ Die Rückstellungen in Höhe von 47 Milliarden Euro für die Beamtenpensionen und Beihilfen fielen dabei höher aus als die Verbindlichkeiten in Höhe von 42 Milliarden Euro ${ }^{68}$.

Die hohe Verschuldung führte 2010 zu der Zinsausgabenquote von knapp acht Prozent in Hessen und rund neun Prozent in Nordrhein-Westfalen und Niedersachsen ${ }^{69}$. Hingegen lag der entsprechende Wert in Bayern und Sachsen nur bei unter beziehungsweise etwas über drei Prozent. Personalund Zinsausgaben erklären bis zur Hälfte das Volumen eines Landeshaushalts. Der zweitgrößte Kostenblock besteht in den Zuweisungen und Zuschüssen, die beispielsweise in Nordrhein-Westfalen 201020 Milliarden Euro betrugen und damit 38 Prozent der Gesamtausgaben ausmachten ${ }^{70}$. Mehr als die Hälfte dieser Zuweisungen und Zuschüsse erhielten die Kommunen, also rund elf Milliarden Euro. Da sich laut der Schuldenbremse die Länder ab 2020 nicht mehr strukturell verschulden dürfen, befürchten die Kommunen, dass genau an dieser Stelle gespart wird. Die offizielle Planung der Länder sieht zwar vor, die Zuwachsraten der Ausgaben deutlich unter diejenigen der Einnahmen zu drücken, um so einen Haushaltsausgleich zu

${ }^{66}$ Vgl. Hamburg zieht Bilanz. Geschäftsbericht 2009, hrsg. von der Freien und Hansestadt Hamburg, Hamburg 2010.

${ }^{67}$ Vgl. Eröffnungsbilanz, hrsg. vom Hessischen Ministerium der Finanzen, Wiesbaden 2009, S. 78. Ein Jahr später, also zum 31. 12.2009, hatte sich das negative Eigenkapital bereits auf 65 Milliarden Euro erhöht. Vgl. Geschäftsbericht 2009, hrsg. vom Hessischen Ministerium der Finanzen, Wiesbaden 2010, S. 63.

${ }^{68}$ Vgl. Eröffnungsbilanz Hessen, S. $78 f$.

${ }^{69}$ Vgl. Jahrbuch für öffentliche Finanzen 2010, S. 103 (Hessen), S. 123 (NordrheinWestfalen) und S. 112 (Niedersachsen) - eigene Berechnungen. Die folgenden Angaben finden sich ebenda, S. 49 (Bayern) und S. 155 (Sachsen) - eigene Berechnungen.

${ }^{70}$ Vgl. Finanzplanung Nordrhein-Westfalen 2009 bis 2013, S. 41; die folgenden Angaben finden sich ebenda. 
erreichen. Dieses Konzept ist allerdings mit großen Risiken behaftet. Ob die Gewerkschaften der Deckelung der Steigerungsraten bei den Personalausgaben zustimmen werden, ist ungewiss. Zudem sieht es derzeit nicht nach einer deutlichen Reduzierung des Personalkörpers aus. So sollen beispielsweise die rückläufigen Schülerzahlen nicht zum Abbau von Lehrerstellen führen, sondern nur zur Verkleinerung der Schulklassen. Ein weiteres Risiko in der Finanzplanung der Länder besteht in der Entwicklung der Zinsausgaben, die förmlich explodieren würden, wenn das Zinsniveau kräftig stiege. Sollten dann noch die Steuereinnahmen nicht konstant oder nur gering steigen $^{71}$, müssten die Länder aufgrund der Schuldenbremse nach Hilfen des Bundes rufen ${ }^{72}$ - und kräftig zulasten der Kommunen sparen ${ }^{73}$. Dieser Mechanismus stellt in der Geschichte eher die Regel als die Ausnahme dar.

\section{Strukturelle Krise der Kommunalfinanzen}

Die auf Johannes von Miquel zurückgehende Finanzreform von 1890/93 war eine erfolgreiche Antwort auf die damalige Krise der Kommunalfinanzen. Sie ermöglichte den preußischen Kommunen mit den Zuschlagsrechten auf die Einkommen-, Gewerbe- und Grundsteuer ein hohes Maß an Finanzautonomie $^{74}$. Die erheblichen Einnahmemöglichkeiten führten zu einer Blüte der kommunalen Selbstverwaltung und legten die Grundlage der städtischen Infrastruktur. 1919 wurde die Finanzautonomie der Kommunen erheblich beschnitten, da sie das Zuschlagsrecht auf die Einkommensteuer verloren. Der Kampf der kommunalen Verbände war während der gesamten Weimarer Zeit darauf abgestellt, dieses Zuschlagsrecht wiederzuerlangen. Immer wieder in Aussicht gestellt, schien dieses Ziel einige Male zwar greifbar nahe zu sein, doch die Weltwirtschaftskrise machte letztlich alle Hoffnungen der Städte und Gemeinden zunichte.

\footnotetext{
${ }^{71}$ Hessen plant beispielsweise mit einem durchschnittlichen Wachstum der Einnahmen 2010-2020 in Höhe von 2,9 Prozent, während die Ausgaben nur um 1,1 Prozent pro Jahr steigen dürfen; vgl. Die Einführung der Schuldenbremse in Hessen. Hintergründe und Argumente, hrsg. vom Hessischen Ministerium der Finanzen, Wiesbaden 2010, S. $12 \mathrm{f}$.

${ }^{72}$ Vgl. Lars P. Feld, Sinnhaftigkeit und Effektivität der deutschen Schuldenbremse, in: Perspektiven der Wirtschaftspolitik 11 (2010), S. 226-245, hier S.239ff.

${ }^{73}$ Vgl. Helmit Dedy/Ann Dahlke, Die Auswirkungen der Schuldenbremse auf die Kommunen, in: Der Gemeindehaushalt 112 (2011), S. 1 ff., hier S. 1.

${ }^{74}$ Vgl. Hermann Elsner, Das Gemeindefinanzsystem. Geschichte, Ideen, Grundlagen, Köln 1979, sowie Hansmann, Kommunalfinanzen.
} 
Mit der Finanzreform von 1969 wurde die Möglichkeit eines kommunalen Einkommensteuerzuschlags zwar ausdrücklich ins Grundgesetz aufgenommen, aber bis heute nicht durch ein Gesetz umgesetzt. Die Kommunen befürchten erhebliche Umverteilungswirkungen ${ }^{75}$, während die Unternehmensverbände vor allem die Gewerbesteuer abschaffen wollen und als Ersatz Zuschläge auf die Einkommen- und Körperschaftsteuer ins Spiel brachten ${ }^{76}$. Im Rahmen der 2010 eingesetzten Gemeindefinanzkommission versuchte Bundesfinanzminister Wolfgang Schäuble (CDU), ein kommunales $\mathrm{Zu}$ schlagsrecht auf die Einkommensteuer durchzusetzen ${ }^{77}$, scheiterte aber am erbitterten Widerstand des Städtetags.

Die Gemeindefinanzreform steht immer dann auf der politischen Agenda ganz oben, wenn die strukturelle Krise der Kommunalfinanzen durch eine Phase des konjunkturellen Abschwungs verschärft wird. Die Kommunen befinden sich seit Anfang der 1990er Jahre in einer Finanzkrise. Seitdem schafften viele Städte und Gemeinden den gesetzlich vorgeschriebenen Haushaltsausgleich nur in Boomjahren. Haushaltsüberschüsse, die im System der kommunalen Haushalte die Investitionen finanzieren sollen, konnten kaum mehr erwirtschaftet werden; im Gegenteil, meist sind die städtischen Haushalte in den letzten 20 Jahren tief defizitär gewesen. Dass diese Defizite nicht nur ein Problem des Kämmerers sind, sondern reale Auswirkungen haben, zeigt der Zustand der kommunalen Infrastruktur ${ }^{78}$. Hier verhält es sich nicht anders als bei einem privaten Haushalt. Wer sein Girokonto permanent überzieht, kann sich keine Investitionen leisten. 1973 standen Investitionen und Sozialausgaben der Stadt Hannover im Verhältnis von drei zu eins zueinander. 37 Jahre später waren die Sozialausgaben acht Mal so hoch wie die Investitionen ${ }^{79}$.

Die wichtigste Kennzahl der Krise ist die Höhe der Kassenkredite. Diese dienen eigentlich nur zur Überbrückung kurzfristiger Liquiditätsengpässe,

\footnotetext{
${ }^{75}$ Vgl. Michael Broer, Wirkungen des kommunalen Zuschlagrechts zur Einkommenund Körperschaftsteuer, in: Wirtschaftdienst 9/2003, S. 599-607.

${ }^{76}$ Vgl. Verfassungskonforme Reform der Gewerbesteuer. Konzept einer kommunalen Einkommen- und Gewinnsteuer, hrsg. vom Bundesverband der Deutschen Industrie/ Verband der Chemischen Industrie, Köln 2001.

${ }^{77}$ Vgl. Werner Gatzer, Reform der Gemeindefinanzen - Ausgabenentlastung allein reicht nicht, in: Hansmann (Hrsg.), Kommunalfinanzen in der Krise, S. 141-153; das Folgende nach diesem Sammelband.

${ }^{78}$ Vgl. Michael Reidenbach u.a., Investitionsrückstand und Investitionsbedarf der Kommunen. Ausmaß, Ursache, Folgen, Strategien, Berlin 2008.

${ }^{79}$ Vgl. die Haushaltspläne der Landeshauptstadt Hannover von 1975 und 2010 (eigene Berechnungen).
} 
stopfen aber seit rund 20 Jahren die Löcher in den kommunalen Haushalten. Während die langfristige Verschuldung der Kommunen verglichen mit Bund und Ländern alles andere als dramatisch erscheint und seit Jahrzehnten mehr oder weniger stagniert, haben viele Städte und Gemeinden ihr Girokonto heftig überzogen. Bereits 2007, also vor der Wirtschaftskrise, waren Kassenkredite in Höhe von fast 30 Milliarden Euro aufgelaufen ${ }^{80}$. Selbst diese Zahl klingt nicht dramatisch hoch. Wer jedoch mittels eines Girokontos einen Teil seiner laufenden Ausgaben finanziert, ist im Grunde bankrott. Die Kommunen haben im Vergleich zu einer Privatperson jedoch zwei Vorteile: Sie sind nicht insolvenzfähig, und sie bekommen kurzfristige Kredite zu extrem günstigen Konditionen.

Die nicht ausgeglichenen Kommunalhaushalte ergeben sich aus der Zangenbewegung von stark steigenden Sozialausgaben und einbrechenden Steuereinnahmen. Mit über 40 Milliarden Euro 2009 haben sich die kommunalen Sozialausgaben seit Anfang der 1990er Jahre beinahe verdoppelt ${ }^{81}$. Hingegen vermochten die meisten Kommunen, den Anstieg ihrer Personalund Sachkosten zu reduzieren. Die Einsparungen reichten jedoch oftmals nur dafür aus, die vom Gesetzgeber neu veranlassten Aufgaben zu finanzieren. Bund und Länder haben das kommunale Ausgabenproblem durch nicht ausreichend finanzierte Aufgabenübertragungen zu einem erheblichen $\mathrm{Ma} \beta$ mit verursacht. Das anschaulichste Beispiel ist die Kindertagesbetreuung. Hier haben sich die Ausgaben der Stadt Hannover von 1989 bis 2009 fast verfünffacht ${ }^{82}$. Möglicherweise wäre der dramatische Anstieg der Sozialausgaben sogar noch aufzufangen gewesen, wenn nicht zur gleichen Zeit die Einnahmenbasis weggebrochen wäre. Das Einnahmenproblem ist jedoch nicht nur die zwangsläufige Konsequenz konjunktureller Abschwungphasen, sondern zu einem großen Teil auch die Folge steuerpolitischer Entscheidungen auf Bundesebene ${ }^{83}$.

Die strukturelle Krise der Kommunalfinanzen, die sich insbesondere im dramatischen Anstieg der kurzfristigen Verschuldung auf über 40 Milliarden Euro Ende 2010 manifestiert, ist hauptsächlich eine Folge des unzureichenden Finanzausgleichs zwischen Bund und Ländern auf der einen Seite und den Kommunen auf der anderen Seite.

\footnotetext{
${ }^{80}$ Vgl. Gemeindefinanzbericht 2008, in: Der Städtetag 5/2008, S. 6.

${ }^{81}$ Vgl. Sozialleistungen der Städte in Not, S. 6.

${ }^{82}$ Vgl. Haushaltspläne der Landeshauptstadt Hannover von 1991 und 2010.

${ }^{83}$ Vgl. Hansmann, Einnahmenproblem, S. 236-242.
} 